\title{
Validez y reproducibilidad de un cuestionario de frecuencia de consumo de calcio para mujeres entre 30 y 50 años
}

Validation and reproducibility of a calcium frequency of consumption questionnaire for 30 through 50 year-old women

\author{
Carlos Rojas ${ }^{1}$, Ivonne Bernui ${ }^{2,3}$, Rosa Oriondo ${ }^{2,3}$, Enriqueta Estrada ${ }^{2}$, \\ Carmen Villarreal ${ }^{2}$, Sissy Espinoza ${ }^{2}$ \\ ${ }^{1}$ Ex Docente de la Facultad de Medicina, UNMSM. Senior Advisor Evaluation, Monitoring and Advocacy Care, Atlanta, EEUU. \\ ${ }^{2}$ Docentes del Departamento de Nutrición, EAP de Nutrición, Facultad de Medicina, Universidad Nacional Mayor de San Marcos. \\ ${ }^{3}$ Centro de Investigación de Bioquímica y Nutrición, UNMSM.
}

\begin{abstract}
Resumen
Objetivos: Medir la validez y la reproducibilidad de un cuestionario de frecuencia de consumo de alimentos (FCA), para evaluar el consumo de calcio en mujeres. Diseño: Estudio de tipo longitudinal y prospectivo. Lugar: Hogares en la ciudad del Lima, del entorno familiar de los estudiantes de la EAP de Nutrición. Participantes: Mujeres de 30 a 50 años. Métodos: Se capacitó 78 mujeres voluntarias entre 30 y 50 años de edad para llevar un registro de alimentos (REG) ingeridos durante 7 días, en dos períodos distintos, con cuatro meses de diferencia entre ambos. En el segundo periodo se les administró una encuesta de FCA con 24 alimentos y preparaciones presentadas en porciones habituales. Para medir la concordancia se calculó la rho de Spearman y el coeficiente kappa; también la sensibilidad y especificidad de la FCA; se elaboró un gráfico de Bland y Altman. Principales medidas de resultados: Miligramos de calcio consumidos, según REG y FCA. Resultados: Los promedios de consumo de calcio durante 14 días en los registros de 24 horas, según FCA1 y el FCA2, fueron $415 \mathrm{mg} \pm 130,4 \mathrm{mg}, 564 \mathrm{mg} \pm 362,5$ y $490 \mathrm{mg} \pm 338,3 \mathrm{mg}$, respectivamente. El coeficiente kappa fue 0,247, bajo pero significativo. El $98,7 \%$ y $76,9 \%$ de las mujeres tuvieron consumo deficiente según los 14 registros y según la FCA, respectivamente. La sensibilidad fue $77,9 \%$, la especificidad $100 \%$ y el valor predictivo positivo $100 \%$. Conclusiones: Los coeficientes de asociación encontrados fueron bajos. Sin embargo, debido a la alta incidencia de consumo deficiente de calcio el formato empleado tiene una buena capacidad de detectar a las personas con un consumo deficiente.
\end{abstract}

Palabras clave: Calcio, frecuencia de consumo, mujeres.

Abstract

Objectives: To determine the validity and reproducibility of a food frequency questionnaire (FFQ) in order to assess calcium intake in women. Design: Longitudinal and prospective study. Location: Homes of Nutrition EAP students' family environment in Lima. Participants: Women volunteers 30 to 50 year-old. Methods: Seventy-eight women volunteers 30 to 50 year-old were trained to keep food records (REC) for 7 days in two distinct periods four months apart. In the second period a FFQ survey with 24 food and preparations in normal portions was applied. Correlation was determined with Spearman rho and Kappa coefficient, as well as FFQ sensitivity and specificity; we present a Bland and Altman graphic. Main outcome measures: Milligrams of calcium consumed by REC and FFQ. Results: Mean calcium intake for 14 days obtained by $24 \mathrm{~h}$ record was $415 \mathrm{mg} \pm 130.4 \mathrm{mg}$ for FFQ1 and $490 \mathrm{mg} \pm 338.3 \mathrm{mg}$ for FFQ2. Kappa coefficient was 0.247 , low, but significant; $98.7 \%$ and $76.9 \%$ of women had inadequate intake based on 14 records of $24 \mathrm{~h}$ and FFQ, respectively. Sensitivity was $77.9 \%$, specificity $100 \%$ and positive predictive value $100 \%$. Conclusions: Correlation coefficients obtained were low, but due to the high incidence of inadequate calcium ingestion the format employed had the ability to detect people with poor consumption.

Key words: Calcium, food frequency questionnaire, women.

An Fac med. 2011;72(3):191-6

\section{INTRODUCCIÓN}

El calcio es conocido por su rol en la formación y manutención del tejido óseo ${ }^{(1)}$; por ello se le ha involucrado en la patogénesis de la osteoporosis, pero también en otras enfermedades crónicas, como la hipertensión, obesidad y el cáncer, enfermedades cuya evolución ocurre a lo largo de varios años ${ }^{(2,3)}$. Cuando se estudia la relación entre dieta y enfermedades crónicas, los estudios tienen necesariamente que referirse al consumo en el largo plazo y habitualmente en el pasado, siendo la herramienta más utilizada en este tipo de estudios el cuestionario de frecuencia de consumo de alimentos. Este ins- trumento es práctico, pues es de fácil uso y requiere un tiempo breve de aplicación, pero necesita ser validado contra otros métodos más exactos, como los registros dietéticos, para probar su efectividad en la población en estudio ${ }^{(3)}$. Las características deseables de un cuestionario de frecuencia de consumo son la habilidad de producir esti- 
mados repetibles y válidos de la ingesta de calcio promedio, así como clasificar correctamente (especificidad y sensibilidad altas) a los individuos en categorías de consumo de calcio ${ }^{(3)}$.

Existen estudios que ya han llevado a cabo validaciones de cuestionarios de frecuencia de consumo de calcio en poblaciones europeas y americanas, los cuales han comparado este con registros dietéticos de 4 días ${ }^{(4)}$ y de 7 días ${ }^{(5,6)}$, habiéndose obtenido resultados diversos, pero siempre tendientes a apoyar el uso de dicha metodología para el desarrollo y validación de cuestionarios de frecuencia de consumo como herramientas epidemiológicas útiles y confiables.

El objetivo del presente trabajo fue medir la validez y reproducibilidad de un cuestionario semicuantitativo de frecuencia de consumo de alimentos (FCA) diseñado en la Universidad $\mathrm{Na}$ cional Mayor de San Marcos, como un instrumento eficaz para detectar diferencias en el consumo de calcio en mujeres entre los 30 y 50 años de edad.

\section{MÉTODOS}

El estudio fue de tipo longitudinal y prospectivo. La población estuvo conformada por mujeres entre los 30 y 50 años de edad. El tamaño de la muestra fue calculado utilizando una fórmula estándar ${ }^{(7)}$ y asumiendo hallar un coeficiente de correlación de 0,5 , con un $\alpha=0,05$ y $\beta=0,8$, y una pérdida del $10 \%$. El tamaño de la muestra resultó 25 mujeres.

$$
\mathrm{n}=\left(\left(\left(\mathrm{Z}_{\alpha}+\mathrm{Z}_{\beta}\right) * 1-\mathrm{r}^{2}\right)^{2} / \mathrm{r}\right)+2
$$

El método de muestreo fue por conveniencia. Participaron en el estudio mujeres de la ciudad de Lima que pertenecían al entorno familiar o social de los investigadores de campo (estudiantes de nutrición de la UNMSM, del tercer año de estudios o más) y que aceptaron participar voluntariamente.

El formato para el registro de consumo de alimentos (REG) fue elaborado

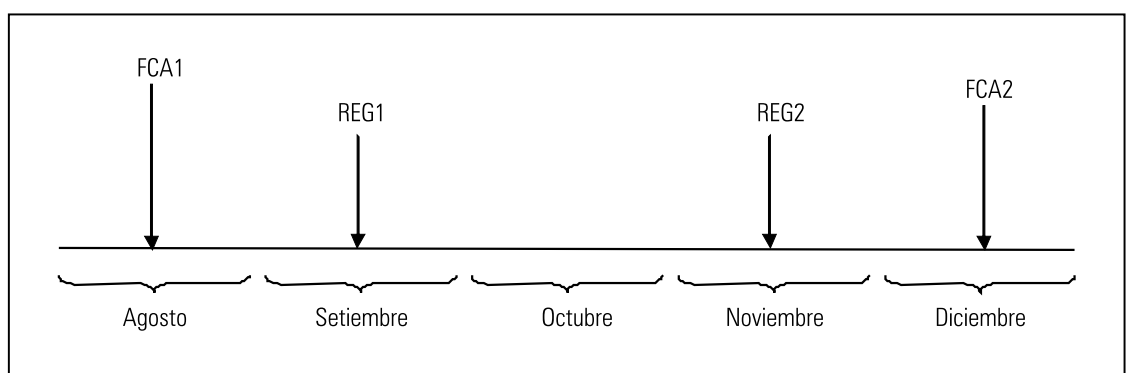

Figura 1. Momentos de aplicación de las encuestas de consumo durante el estudio.

a partir de un modelo estándar ${ }^{(8)}$, en el cual se registró el nombre del alimento, la medida casera y la cantidad de medidas caseras utilizadas.

La encuesta semicuantitativa de frecuencia de consumo de alimentos (FCA) estuvo conformada por un listado de 12 alimentos y 13 preparaciones presentadas en porciones habituales de consumo y un conjunto de categorías de frecuencias de consumo diario, semanal y mensual, subdivididas en porciones por día, semana o mes Los alimentos que conformaron la lista fueron obtenidos de un estudio previo ${ }^{(9)}$.

La FCA fue aplicada a cada una de las mujeres participantes en el estudio en dos momentos, con cuatro meses de diferencia, con la finalidad de comprobar si los datos obtenidos por la FCA eran reproducibles en el tiempo (figura 1). Los REG fueron recogidos en dos períodos de 7 días cada uno, siendo en total 14 días de información.

El registro del consumo de alimentos fue elegido como el método estándar de comparación, porque a diferencia del recordatorio de 24 horas, no depende de la memoria ${ }^{(8)}$. Tal y como indica el método, las mismas mujeres se encargaron de llenarlo a lo largo del día, para lo cual fueron instruidas por los investigadores de campo ${ }^{(8)}$. Al final de cada día, los investigadores de campo verificaron los registros de alimentos para asegurar la consistencia de los mismos.

El cuestionario de FCA fue aplicado a la población en estudio por 36 investigadores de campo, registrando la fre- cuencia de consumo de los alimentos según número de porciones consumidas en un tiempo determinado.

Antes del procesamiento de los datos se realizó una limpieza de los mismos. Posteriormente, fueron introducidos a las bases de datos electrónicas diseñadas con la hoja de cálculo Excel 97 (RCA) y Epi Info 6,04 b (FCA).

La determinación de los miligramos (mg) de calcio consumido se realizó tanto a partir del registro de consumo de alimentos (REG) como a partir del cuestionario semicuantitativo de frecuencia de consumo de alimentos (FCA). Por considerarse que 14 días reflejarían mejor el consumo habitual que 7 días, se promedió los resultados obtenidos por ambos registros en una nueva variable (REG).

El consumo de calcio se presenta como media con desviación estándar, mediana e intervalo intercuartílico. Se calculó la rho de Spearman. Así mismo, se dividió los resultados de cada uno de los métodos en cuartiles y luego se calculó el valor de kappa (índice de concordancia). Se usó la formula de Cohen, citado por Fleiss ${ }^{(10)}$, quien propuso la siguiente:

$$
\mathrm{K}=\frac{\mathrm{po}-\mathrm{pc}}{1-\mathrm{pc}}
$$

Donde:

- po = es la proporción de las celdas centrales entre $\mathrm{n}^{2}$.

- $\mathrm{pc}=$ es la proporción calculada de las celdas centrales según el azar. 
Tabla 1. Estadísticas descriptivas de registro de consumo (REG) y frecuencia de consumo (FCA1 y FCA2).

\begin{tabular}{|c|c|c|c|}
\hline \multicolumn{2}{|c|}{ FCA-1 } & FCA-2 & REG \\
\hline N & 78 & 78 & 78 \\
\hline Media & 564,6 & 490,0 & 415,8 \\
\hline Desviación estándar & 362,5 & 338,3 & 130,4 \\
\hline Mediana & 449,0 & 278,3 & 418,0 \\
\hline Rango intercuartílico & 333 a 753 & 392 a 625 & 320 a 491 \\
\hline
\end{tabular}

Tabla 2. Reproducibilidad del formato de frecuencia de consumo aplicado en dos momentos.

\begin{tabular}{|c|c|c|c|c|c|}
\multirow{2}{*}{$\begin{array}{c}\text { Segunda } \\
\text { encuesta, FCA2 }\end{array}$} & 01 & 02 & 03 & Total \\
\hline 01 & 13 & 2 & 2 & 3 & 20 \\
\hline 02 & 2 & 10 & 3 & 4 & 19 \\
\hline 03 & 2 & 4 & 7 & 6 & 19 \\
\hline 04 & 3 & 3 & 7 & 7 & 20 \\
\hline Total & 20 & 19 & 19 & 20 & 78 \\
\hline
\end{tabular}

Se elaboró un gráfico de Bland-Altman, técnica gráfica usada para comparar dos métodos de cuantificación de la misma variable ${ }^{(11)}$. Se utilizó el software SPSS para llevar a cabo las pruebas estadísticas con un nivel de confianza de $95 \%$.

\section{RESULTADOS}

Se analizó la distribución de las variables mg de calcio obtenido con 14 dias

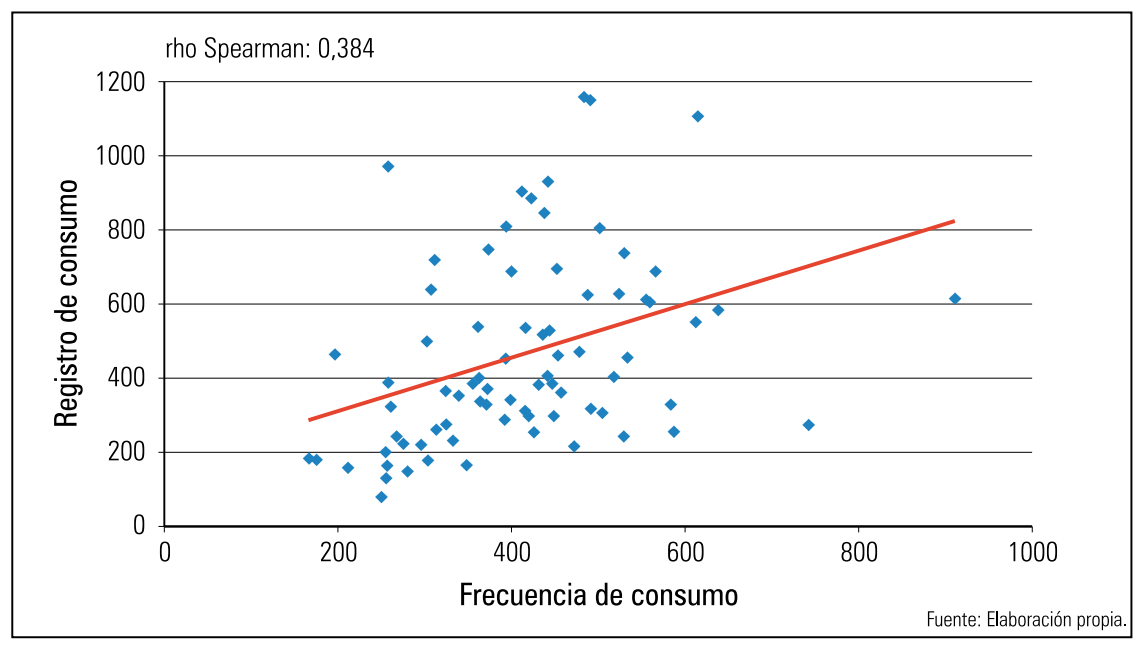

Figura 2. Diagrama de dispersión para registro de consumo y frecuencia de consumo.
Se hizo un diagrama de puntos (figura 2) entre el promedio de consumo de calcio según REG y FCA, observándose una gran dispersión de los datos. El coeficiente rho Spearman fue 0,384, lo que indicó una asociación baja entre ambas metodologias de evaluación del consumo de calcio.

Para estimar la reproducibilidad, se clasificó a la población en cuartiles, según los datos obtenidos en la primera encuesta de frecuencia de consumo como en la segunda (tabla 2). Se esperaba que si ambas pruebas fueran concordantes se debió tener en las celdas centrales una mayor frecuencia que en las celdas adyacentes. Se encontró que $78 \%$ de los datos de la muestra caía en el mismo cuartil o en uno adyacente, lo cual nos indicaba un cierto grado de asociación, porque estaba por encima del valor que se hubiese obtenido por el azar (62,5\%).

Para medir la validación, se clasificó a la población en cuartiles, según los resultados del registro de consumo de 14 dias (REG) y la frecuencia de consumo (FCA1), para determinar si los datos caían en los mismos cuartiles según cada una de las pruebas. El $81 \%$ de los datos cayó en la celda central o en una adyacente y solo $4 \%$ en celdas opuestas, lo cual nos indicó una asociación regular, que estaba $18,5 \%$ por encima de las coincidencias esperadas por el azar $(62,5 \%$, tabla 3$)$. Se realizó la prueba kappa de Cohen para conocer la concordancia de ambos métodos y se obtuvo un valor de 0,247 , lo cual indicó una asociación baja, aunque significativa $(\mathrm{p}<0,05)$.

Los percentiles de consumo de calcio con cada uno de los métodos estuvieron cercanos hasta el $50^{\circ} \mathrm{P}$; a partir de allí, los resultados de la frecuencia de consumo fueron más altos y la diferencia fue aumentando desde el 50ㅇ al 100 ㅇ (figura 3).

Se consideró el requerimiento de calcio para este grupo etáreo $(800 \mathrm{mg})$ como punto de corte, para determinar quiénes tenían un consumo bajo y así poder diferenciar los consumos adecua- 
Tabla 3. Comparación de la distribución de personas según cuartiles por frecuencia de consumo de alimentos y por registro (REG) de 14 días.

\begin{tabular}{|c|c|c|c|c|c|}
\hline \multirow{2}{*}{$\begin{array}{c}\text { Registro de consumo } \\
\text { de alimentos, REG }\end{array}$} & C1 & C2 & C3 & C4 & Total \\
\hline C1 & 14 & 3 & 2 & 1 & 20 \\
\hline C2 & 1 & 10 & 4 & 4 & 19 \\
\hline C3 & 3 & 3 & 4 & 9 & 19 \\
\hline C4 & 2 & 3 & 9 & 6 & 20 \\
\hline Total & 20 & 19 & 19 & 20 & 78 \\
\hline
\end{tabular}

Tabla 4. Distribución de muestra en consumo de calcio adecuado e inadecuado.

\begin{tabular}{|c|c|c|c|}
\hline \multirow{2}{*}{ FCA1 } & \multicolumn{2}{|c|}{ REG } & \multirow{2}{*}{ Total } \\
\hline & Inadecuado & Adecuado & \\
\hline Inadecuado & 60 & 0 & 60 \\
\hline Adecuado & 17 & 1 & 18 \\
\hline Total & 77 & 1 & 78 \\
\hline
\end{tabular}

dos e inadecuados según cada uno de los métodos (tabla 4).

A partir la tabla 4 se calculó la sensibilidad (78\% para detectar a los sujetos con consumo inadecuado) y la especificidad (100\% para detectar a la personas con consumo adecuado), para el formato de frecuencia de consumo. Sin embargo, la especificidad obtenida debiera ser tomada con mucha cautela, dado que se trata de un solo dato (1\% de la muestra), y esto simplemente re-

fleja el consumo bajo de calcio de este grupo etáreo, que a su vez es semejante al de otros grupos en el Perú ${ }^{(5)}$.

Se hizo un gráfico Bland-Altman entre el promedio (eje $\mathrm{X}$ ) y la diferencia (eje Y) de los mg de calcio consumidos, según cada uno de los métodos. Se pudo observar que varios datos cayeron por debajo de una desviación estándar de la diferencia de ambos métodos (figura 4).

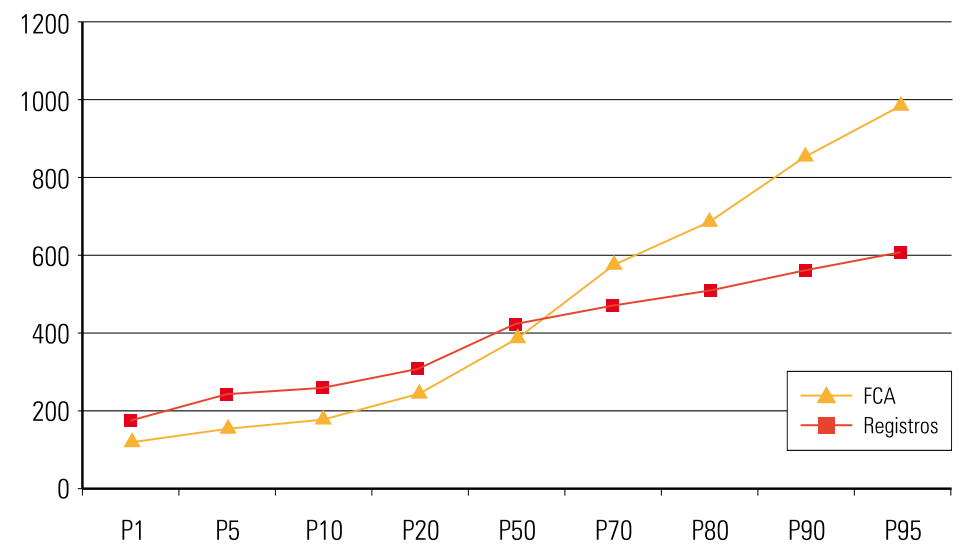

Figura 3. Comparación de los percentiles de consumo según frecuencia de consumo de alimentos y según registro de consumo.

\section{DISCUSIÓN}

Todavía no existe un consenso entre los expertos del área de validación de encuestas de consumo acerca de la manera 'estándar' de presentar la información sobre estudios de validación. Como reconocen Margetts y Thompson ${ }^{(11)}$, no existe por el momento (y se duda mucho que alguna vez exista en el futuro) una verdadera 'prueba de oro' contra la cual deban medirse los métodos rápidos, por lo cual señalan que estos estudios son de una validez relativa y que debieran más bien llamarse 'estudios de calibración', para describir el proceso de determinar si existe concordancia adecuada entre dos métodos que se cree tienen errores independientes, pero que miden la misma cosa. También, reconocen que la correlación de rangos de Spearman como el kappa de Cohen serían alternativas apropiadas a ser usadas en distribuciones sesgadas. Sin embargo, el inconveniente de estos índices es que estarían reflejando las características de la población de estudio. Por ejemplo, si existe una gran variación en la ingesta entre los individuos es más fácil distinguir entre sujetos que en un estudio con un grupo más homogéneo, y esto se reflejaría en valores más altos de correlación. Sugieren dichos autores que se debería usar medidas de dispersión de las diferencias entre los pares de observación reportados, de manera tal que se reflejen las características del método y no de la población en estudio.

Una de las características que debe tener un instrumento de evaluación debe ser la repetibilidad. $\mathrm{Al}$ realizar por segunda vez la encuesta de frecuencia de consumo de alimentos (FCA) encontramos resultados similares, lo cual nos asegura que tendremos precisión al momento de usarlo. Sin embargo, el coeficiente de correlación hallado en el presente estudio ha estado por debajo de lo reportado por Wilson y Horwath ${ }^{(5)}$, quienes obtuvieron un $r=0,81$.

Hemos podido comprobar que $81 \%$ de las mujeres encuestadas coincidió en el mismo quintil o en uno adyacente; 


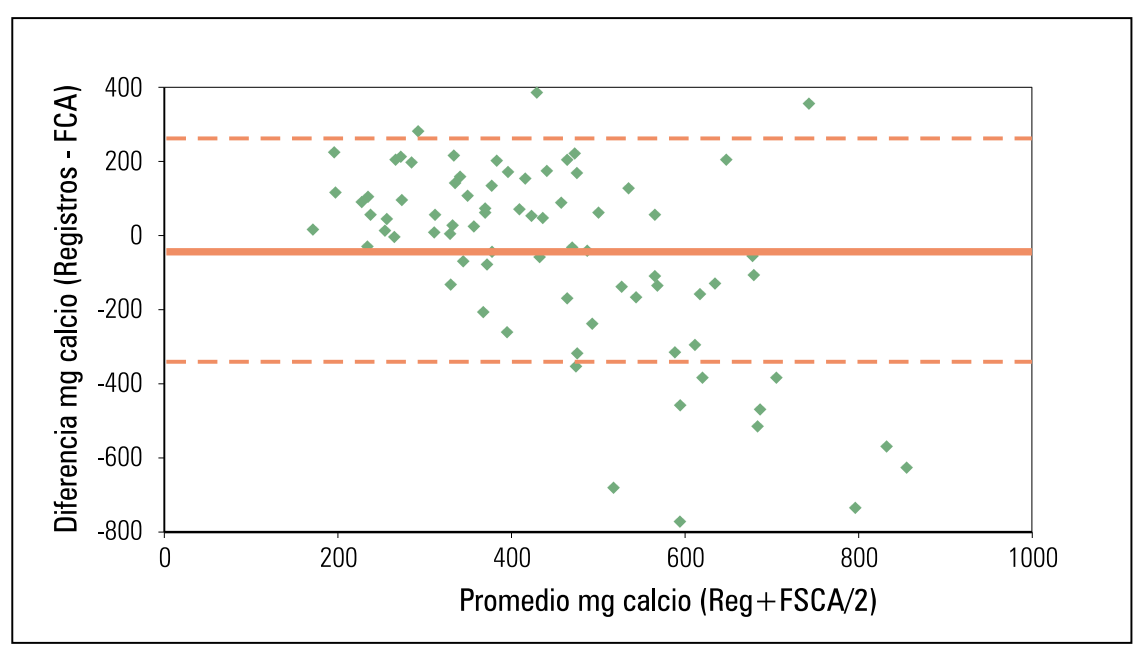

Figura 4. Comparación Bland-Altman del promedio de mg de calcio por ambos métodos (eje X), con la diferencia de mg de calcio por ambos métodos (eje Y).

este porcentaje es mayor al que se obtendría si la clasificación fuera al azar (65\%). Este valor es igual al encontrado por Wilson y Harworth ${ }^{(5)}$.

Block y Hartman ${ }^{(14)}$ señalan los diferentes factores a tener en cuenta en los estudios de validación: las características de los encuestados, el diseño del cuestionario y su cuantificación, adecuación de los datos de referencia y el control de calidad en el manejo de los datos. El formato del presente estudio podría haber dado lugar a escribir la respuesta en el renglón equivocado y ese aspecto del trabajo se podría mejorar.

En cuanto a la lista de alimentos incluidos, Block y Hartman ${ }^{(14)}$ señalan que no puede ser tan corta que se omita importantes fuentes de nutrientes para la población estudiada, ni tampoco muy larga que produzca 'ruido' al hacer los estimados. En el presente estudio, a pesar que el registro de consumo cubrió un mayor número de alimentos y por tanto el 100\% del calcio consumido, los promedios obtenidos con la frecuencia de consumo han sido más altos, tanto en la primera como en la segunda aplicación del formato, que los promedios calculados en base al registro de consumo.
Los datos de referencia considerados en el presente estudio como representantes del verdadero consumo han sido los registros de consumo de 7 días, repetidos en dos ocasiones. Se considera que al ser un método según el cual la persona va registrando su consumo de alimentos según este ocurre, tendrá una distribución aleatoria de errores diferente al método de frecuencia de consumo, que es un método basado en la memoria. Según señalan los autores, el número de días necesarios para reflejar la ingesta usual a largo plazo dependerá del nutriente que esté en estudio; para el caso de micronutrientes, son necesarios como mínimos 7 a 10 días ${ }^{(3)}$.

En el presente estudio se ha cumplido con el entrenamiento adecuado de los entrevistadores, que al ser tan numeroso, cabría la posibilidad de que cada uno interprete los formatos a su manera.

Block y Hartman ${ }^{(14)}$ encuentran que los coeficientes obtenidos de diferentes estudios de validación están entre 0,5 y 0,6 (o $\geq 0,7$, cuando se corrige la variabilidad de los datos de referencia) y se preguntan si estos valores son adecuados para los estudios que relacionen dieta y enfermedad. Señalan que este rango de correlaciones es el mismo en- contrado en varias medidas fisiológicas (colesterol sérico, presión arterial en adultos, ácido úrico, sodio en 24 horas), debido a la variabilidad intraindividual de dichas medidas.

Según los resultados obtenidos por los diferentes indicadores utilizados, se desprende que el formato propuesto de frecuencia de consumo no puede ser usado de manera intercambiable con los registros de alimentos, si se quiere un estimado del consumo de calcio individual.

Se concluye que, a pesar de haber encontrado coeficientes bajos de asociación, sin embargo, debido a la incidencia alta de consumo deficiente de calcio el formato empleado tiene una buena capacidad de detectar a las personas con un consumo deficiente.

\section{REFERENCIAS BIBLIOGRÁFICAS}

1. Heaney RP. Calcium, dairy products and osteoporosis. J Am Coll Nutr. 2000;19(suppl 2):83S-99S.

2. Power ML, Heaney RP, Kalkwarf HJ, Pitkin RM, Repke JT, Tsang RC, Schulkin J. The role of calcium in health and disease. Am J Gynecol Obstet. 1999;181:1560-9.

3. Heaney RP, Davies KM, Barger-Lux MJ. Calcium and weight: clinical studies. J Am Coll Nutr. 2002;21:S152-5.

4. Willet W. Nutritional Epidemiology. 2nd edit. New York: Oxford University Press, 1998.

5. Musgrave KO, Giambalvo L. Leclere HI, Cook R.A. Validity of quantitive food frequency questionnaire for rapid assessment of dietary calcium intake. J Am Dietetic Assoc. 1989;89(10):1484-8.

6. Wilson P, Horwarth C. Validation of a short food frequency questionnaire for assessment of dietary calcium intake in woman. Eur $\mathrm{J}$ Clin Nutr. 1996;50:220-8.

7. Cummings SR, Block G, Mc Henry K, Baron RB. Evaluation of two frequency questionnaires of measuring dietary calcium intake. Am J Epidemiol. 1987;126(5):796-802.

8. Norman G, Streiner D. Bioestadistica. Barcelona: Editorial Hartcourt. 1996.

9. Gibson RS. Principles of Nutritional Assessment. 2nd edit. New York: Oxford University Press, 2005.

10. Bernui I, Rojas C, Oriondo R y col. Consumo de calcio de los estudiantes de la Universidad Nacional Mayor de San Marcos. Primeras Jornadas de Investigación en Salud. Lima, 14-19 de junio de 1999. Facultad de Medicina. Unidad de Investigación. Universidad Nacional Mayor de San Marcos.

11. Fleiss J. Statistical methods for rates and proportions. New York: John Wily and Sons. 1971.

12. Bland JM, Altman DG. Statistical methods for assessing agreement between two methods of clinical measurement. Lancet. 1986;1:307-10. 
13. Burema J, van Staveren WA \& Feunekes GIJ. Guidelines for reports on validation studies. Letter to the Editor. Eur J Clin Nutr. 1995;49:932-3.

14. Margetts BM, Thompson RL. Validation of dietary intake. Letter to the Editor. Eur J Clin Nutr. 1995;49:934.

15. Block G, Hartman A. Issues in reproducibility and validity of dietary studies. Am J Clin Nutr. 1989;50:1133-8.
16. Freedman L, Carroll RJ, Wax Y. Estimating the relation between dietary intake obtained from a food frequency questionnaire and true average intake. Am J Epidemiol. 1991;134:310-20.

17. Montomoli M, Gonnelli S, Giacchi M, Mattei R, Cuda C, Rossi S, Gennari C. Validation of a food frequency questionnaire for nutritional calcium intake assessment in Italian women. Eur J Clin Nutr. 2002;56:21-30.
Financiamiento: Autofinanciado

Conflicto de intereses: Ninguno

\section{Correspondencia:}

Ivonne Bernui Leo

Correo electrónico: ibernuil@unmsm.edu.pe 